\title{
Diabetic retinopathy: current understanding, mechanisms, and treatment strategies
}

\author{
Elia J. Duh, ${ }^{1}$ Jennifer K. Sun, ${ }^{2}$ Alan W. Stitt ${ }^{3}$ \\ 'Wilmer Ophthalmologic Institute, Johns Hopkins University School of Medicine, Baltimore, Maryland, USA. \\ ${ }^{2}$ Joslin Diabetes Center, Harvard Medical School, Boston, Massachusetts, USA. ${ }^{3}$ Centre for Experimental Medicine, \\ Queen's University Belfast, Northern Ireland, United Kingdom.
}

\begin{abstract}
Diabetic retinopathy (DR) causes significant visual loss on a global scale. Treatments for the vision-threatening complications of diabetic macular edema (DME) and proliferative diabetic retinopathy (PDR) have greatly improved over the past decade. However, additional therapeutic options are needed that take into account pathology associated with vascular, glial, and neuronal components of the diabetic retina. Recent work indicates that diabetes markedly impacts the retinal neurovascular unit and its interdependent vascular, neuronal, glial, and immune cells. This knowledge is leading to identification of new targets and therapeutic strategies for preventing or reversing retinal neuronal dysfunction, vascular leakage, ischemia, and pathologic angiogenesis. These advances, together with approaches embracing the potential of preventative or regenerative medicine, could provide the means to better manage DR, including treatment at earlier stages and more precise tailoring of treatments based on individual patient variations.
\end{abstract}

Conflict of interest: The authors declare that no conflict of interest exists.

License: This work is licensed under the Creative Commons Attribution 4.0 International License. To view a copy of this license, visit http:// creativecommons.org/licenses/by/4.0/.

Published: July 20, 2017

Reference information: JCI Insight. 2017;2(14):e93751. https://doi.org/10.1172/jci. insight.93751.

\section{The clinical challenge of diabetic retinopathy}

The global prevalence of diabetes mellitus is predicted to increase dramatically in the coming decades, from an estimated 382 million in 2013 to 592 million by 2035 (1, 2). Type 2 diabetes (T2D) in particular has already attained epidemic levels, while type 1 diabetes (T1D) is increasing in incidence (3). Patients with diabetes suffer many life-limiting and life-threatening complications, including macrovascular-related stroke, ischemic heart disease, and peripheral artery disease and/or microvascular-related retinopathy, neuropathy, and nephropathy. Diabetic retinopathy (DR) is the most common microvascular complication of diabetes (4). Although some reports suggest that the incidence of visual impairment from DR has decreased in recent years in the US largely due to improvements in systemic control (5), DR is a burgeoning problem globally. DR currently affects almost 100 million people worldwide and is set to become an ever-increasing health burden, with estimates between 1990 and 2010 showing that DR-related visual impairment and blindness increased by $64 \%$ and $27 \%$, respectively (6).

\section{DR classification and risk factors}

Based on their obvious manifestations during DR progression, microvascular lesions have been utilized as the major criteria for evaluating and classifying the retina in DR. However, diabetes-induced changes also occur in nonvascular cell types that play an important role in the development and progression of DR, albeit in unison with the vasculature. DR falls into 2 broad categories: the earlier stage of nonproliferative diabetic retinopathy (NPDR) and the advanced stage of PDR. Classification of NPDR is based on clinical findings manifested by visible features, including microaneurysms, retinal hemorrhages, intraretinal microvascular abnormalities (IRMA), and venous caliber changes (Figure 1), while PDR is characterized by the hallmark feature of pathologic preretinal neovascularization (3). While these visible features of DR provide useful measures for detection and diagnosis, improving technology has enabled the detection of more subtle pathologies such as retinal function deficits and neural layer abnormalities in patients $(7,8)$. An important additional categorization in DR is diabetic macular edema (DME), which is an important manifestation of DR that occurs across all DR severity levels of both NPDR and PDR and represents the most common cause of vision loss in patients with DR. DME arises from diabetes-induced breakdown of 
the blood-retinal barrier (BRB), with consequent vascular leakage of fluid and circulating proteins into the neural retina $(3,9,10)$. The extravasation of fluid into the neural retina leads to abnormal retinal thickening and often cystoid edema of the macula.

Many systemic features of diabetes influence DR. For example, hyperglycemia is inextricably linked to DR as evidenced by seminal large-scale clinical trials. The Diabetes Control and Complications Trial (DCCT) for T1D (11) and the United Kingdom Prospective Diabetes Trial (UKPDS) for T2D (12) support intensive glycemic control, as assessed by hemoglobin A1c (HbA1c), to delay initiation and progression of this complication, although the need to avoid hypoglycemia can make intensive control challenging for many patients. The importance of glycemia management as early as possible during the course of diabetes is emphasized by robust preclinical and clinical evidence that indicate the long-term impact of intensive glycemic control. The Epidemiology of Diabetes Intervention and Complications (EDIC) study was an observational follow-up of the DCCT cohort of individuals that had initially received either intensive glycemic control or conventional therapy. Although both groups subsequently underwent intensive glycemic control in the subsequent years encompassed by the EDIC study, the group receiving intensive control during DCCT continued to exhibit a significantly lower incidence of further progression of their diabetic retinopathy severity stage. This durable impact of initial intensive control has been termed metabolic (or, sometimes, glycemic) memory (13), and in DCCT/EDIC patients, there is associative evidence of accumulation of certain advanced glycation endproduct (AGE) adducts in long-lived proteins that correlate with retinopathy risk (14). Whether hyperglycemia alone accounts for the persistent effects of poorly controlled diabetes remains an important question, but nevertheless, the concept of metabolic memory remains an area of intensive investigation. The memory phenomenon has support from studies using animal models of diabetic retinopathy that are returned to normoglycemia (achieved using insulin therapy). For example, hyperglycemia-mediated oxidative damage (15) impaired function of key transcription factors (16), and changes to enzymes controlling the electron transport chain are sustained in the retinas of animals even following several months normoglycemia. Many of these pathways can become dysregulated following DNA and histone methylation, and there is now convincing preclinical evidence that such epigenetic modifications are associated with the metabolic memory phenomenon for not only retinopathy, but also other complications (17). Interestingly, a recent transcriptomics study in the retinas of diabetic mice receiving insulin-producing islet cell transplants has suggested that gene changes relating to metabolic memory may be particularly associated with the neurovascular unit (18).

Dyslipidemia and hypertension may also influence DR (19), although in the context of individual patients, the associations between plasma lipids, lipoproteins, and DR are not sufficiently strong to define retinopathy risk. Likewise, hypertension has been linked to increased risk of DR (20), and some data indicate that patients may benefit from the use of antihypertensive agents (21). However, recent studies have demonstrated that more intensive blood pressure control does not confer additional benefits on retinopathy progression compared with standard control (22). Taken together, optimization of systemic risk factors is clearly important; however, even hyperglycemia (as measured by HbA1c) may only account for around $10 \%$ of DR risk (23), and hypertension and dyslipidemia combined may carry $<10 \%$ risk in some cohorts (24). Such data strongly suggest that additional unidentified factors also play critical roles in DR initiation and progression.

\section{Retinal vascular lesions and pathogenic sequelae of diabetes}

The hallmark microvascular features of NPDR (Figure 1) include intraretinal hemorrhages, microaneurysms, venous caliber abnormalities, formation of IRMA, lipid exudates from the damaged vasculature, capillary nonperfusion with accompanying neuronal infarcts represented as cotton-wool spots, and retinal neovascularization. Retinal vascular lesions, which have been well-characterized histologically in postmortem human eyes and in some long-term preclinical models, are preceded and/or accompanied by vascular basement membrane thickening, an early loss of pericytes, and eventual death of endothelial cells that underpin the vasodegenerative pathology that is a feature of early DR (refs. 25-28; reviewed by refs. 29, 30). Several retinal vascular pathologic processes in DR have a direct impact on vision. In NPDR, gradual nonperfusion of the retinal vascular bed, characterized by loss of vessel integrity, ultimately leads to occlusion or degeneration of capillaries (3). Localized capillary nonperfusion results in regions of ischemia and impaired oxygenation of the metabolically demanding retinal neurons. Progressive capillary nonperfusion and resultant ischemia underpin progression to PDR, which is driven by hypoxia and expression of proangiogenic growth factors, which stimulate the aberrant formation of new blood vessels in the retina that protrude into the preretinal space. Retinal neovascularization can result 
A

\section{Normal Retina}

Severe NPDR with DME

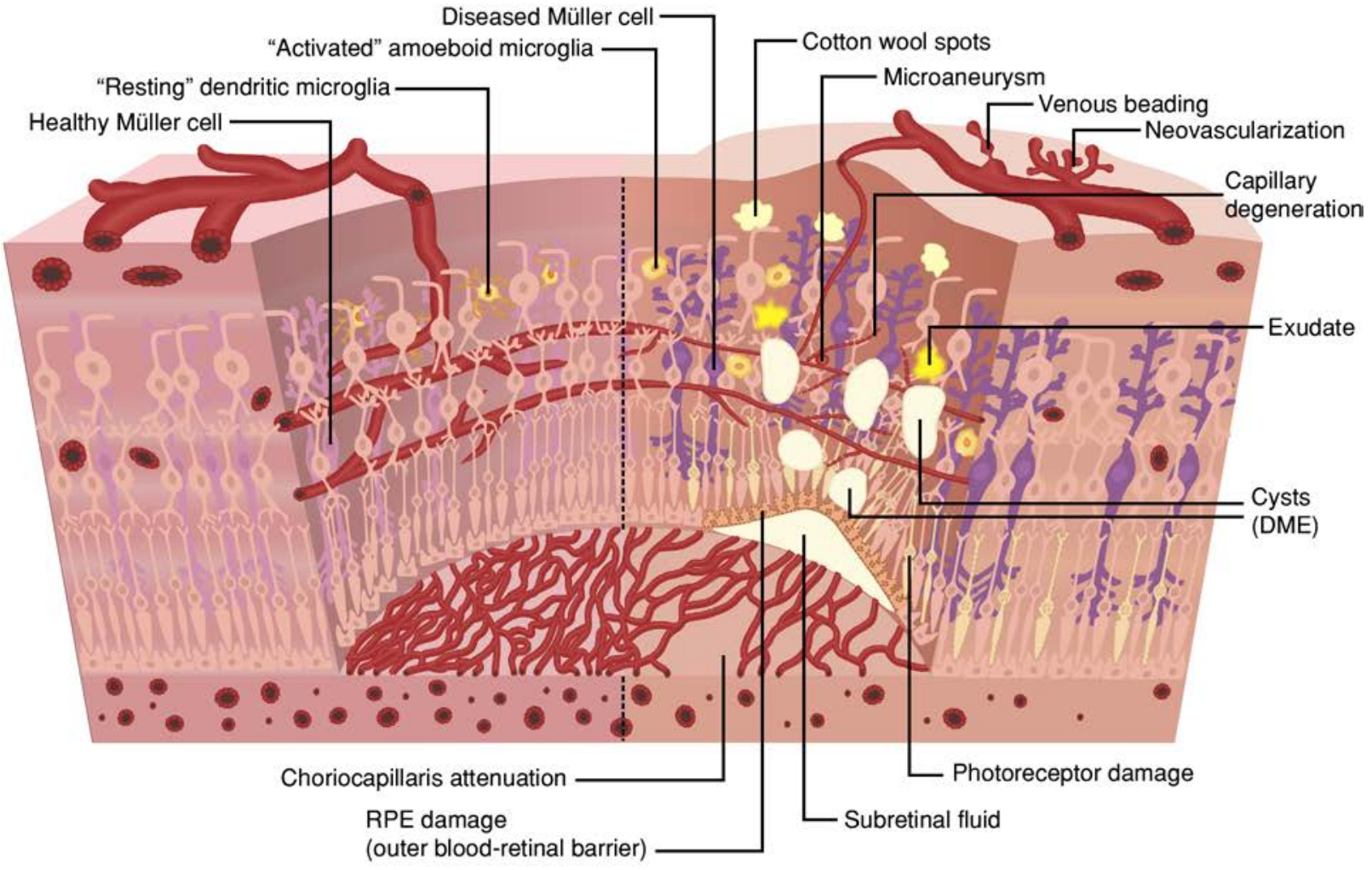

B

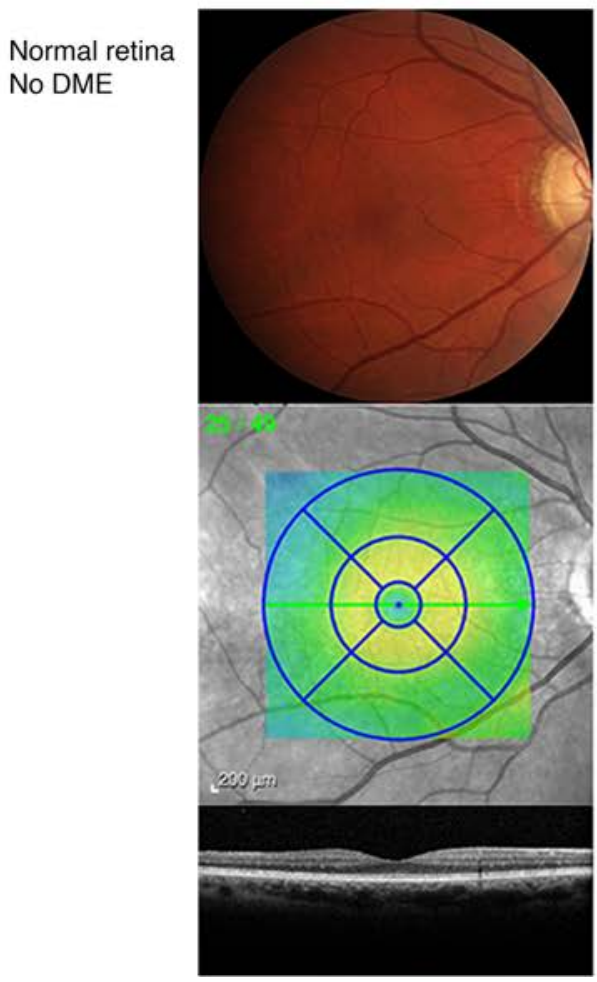

Severe NPDR

Central-involved DME

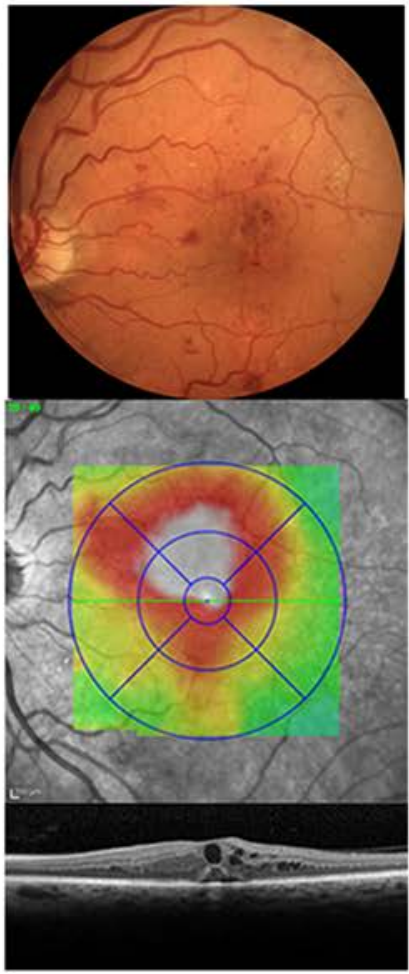

Figure 1. Pathological lesions of diabetic retinopathy. (A) An illustrated schematic of normal retina compared with nonproliferative diabetic retinopathy (NPDR) with diabetic macular edema (DME). The normal healthy retina includes healthy retinal blood vessels, glial elements including Müller cells, neuronal elements including photoreceptors, and resting microglia. The inner and outer blood-retinal barriers are intact. In contrast, the retina in diabetic retinopathy exhibits multiple abnormalities, including vascular changes (microaneurysms, venous beading, capillary degeneration, and neovascularization), 
lesions associated with vascular damage (cotton wool spots and exudate), glial dysfunction including Müller cell swelling, neuronal damage, activated microglia, retinal pigment epithelium (RPE) damage, and thinning of the choriocapillaris. There is dysfunction of the inner and outer blood-retinal barrier, with resulting accumulation of fluid in the retina, which can be manifested by thickening of retinal layers, cysts, and subretinal fluid. (B) Color fundus photograph and OCT images of a normal healthy retina and retina with severe NPDR with central-involved DME are shown. Illustrated by Rachel Davidowitz.

in severe vision loss when it leads to vitreous hemorrhage or tractional retinal detachment (3). Another major pathologic process is DME, which is characterized by overt breakdown of the BRB that leads to macular edema and swelling of the neuropile, which frequently leads to vision loss.

A long-standing mystery in DR and other ischemic retinopathies is the striking lack of revascularization of ischemic retina, despite the strong hypoxia stimulus and enhanced production of proangiogenic growth factors. Indeed, the diabetic milieu within the retina seems to be unfavorable for reparative angiogenesis, possibly due to pathogenic factors such as AGEs (3). More recently, evidence has emerged supporting a possible role for semaphorins, a class of proteins originally implicated in axonal growth cone guidance. Some semaphorin molecules regulate angiogenesis, and several semaphorins - including semaphorin 3A (31), semaphorin 3F (32), and semaphorin 6A (33) - are specifically implicated in suppressing the revascularization response in the ischemic retina, redirecting neo-vessels toward the vitreous instead. As the proangiogenic factors in DR lead almost exclusively to pathologic preretinal neovascularization rather than beneficial revascularization, extensive efforts have been made over decades to identify the major proangiogenic growth factors in PDR, such as VEGF (34). As a result, anti-VEGF treatments have emerged as an effective approach for treatment of this condition $(35,36)$, although many additional proangiogenic pathways will likely also serve as therapeutic targets, including placental growth factor (37), stromal-derived factor-1 (38), and erythropoietin (39). Improvements in understanding the molecular basis of both pathologic retinal neovascularization and deficient revascularization may produce new therapeutic targets that can suppress aberrant angiogenesis in favor of revascularization.

\section{Evaluating the diabetic retina}

Although 7-standard field color fundus photography based on the Early Treatment Diabetic Retinopathy Study (ETDRS) protocol (40) has been the validated standard for evaluation of DR for decades, substantial advances in ocular imaging over the last 2 decades have provided new insights into diabetic vascular and neuroretinal pathology. Ultrawide-field color fundus photographs and fluorescein angiograms can now capture $200^{\circ}$ (or over $80 \%$ ) of the retina in a single image, leading to a better understanding of peripheral changes in the diabetic retina. Indeed, the presence and severity of peripheral DR lesions is predictive of future rates of DR worsening (41). Retinal photographs that utilize adaptive optics technology to compensate for wavefront aberrations in individual eyes allow imaging with a theoretical resolution limit down to 2 $\mu \mathrm{m}$ and have greatly expanded the ability to visualize the retina on a cellular level. Adaptive optics studies demonstrate changes in the cone photoreceptor mosaic in the diabetic eye (42) and allow visualization of early vascular changes that cannot be identified on standard photographs (43).

Optical coherence tomography (OCT) is a widely utilized option for imaging the diabetic neural retina that uses light interferometry to create cross-sectional images of the retina in which individual retinal layers can be distinguished. OCT allows quantitative measurements of retinal thickness, as well as evaluation of morphologic changes in eyes with DR and DME. Potential neuroretinal biomarkers of visual acuity in eyes with DME based on OCT imaging have been suggested, including ganglion cell layer thinning, disorganization of the retinal inner layers, and photoreceptor disruption, although further validation is needed (44-47). Recently, the technique of OCT angiography has also been utilized to create high-resolution perfusion maps of the central retinal vasculature (48).

Both full-field and multifocal electroretinography (ERG) demonstrate abnormalities in retinal electrical signaling in the diabetic eye. Local changes in multifocal ERG implicit time appear to precede the development of DR lesions such as microaneurysms (49). Assessments of visual function demonstrate abnormalities in contrast sensitivity, color testing, frequency-doubling perimetry, and microperimetry in the diabetic eye with varying levels of DR severity; however, these functional tests are not sensitive or specific enough to serve as a reliable surrogate or predictive marker of DR or DME.

Future efforts in DR imaging may be geared toward multimodal evaluation of the retina. The use of simultaneous or near-simultaneous imaging methods that focus on specific components of neural or vascular retina may improve understanding of which pathologies develop first in the diabetic eye and may provide predictive biomarkers of future visual function outcomes in DR. 


\section{Current treatment options and limitations}

Intraocular treatment modalities for diabetic eye disease include laser photocoagulation, intravitreous injections of anti-VEGF and steroid agents, and vitreoretinal surgery. Current therapeutic paradigms focus on treatment of advanced disease, once PDR or DME has developed.

Panretinal photocoagulation (PRP) for PDR was first proposed in the 1960s. Despite initial skepticism that the creation of thermal burns throughout the retinal periphery could promote regression of retinal neovascularization, the efficacy of PRP in reducing rates of severe vision loss in eyes with PDR was quickly and incontrovertibly demonstrated by the nationwide, multicenter Diabetic Retinopathy Study (50). The ETDRS subsequently revealed that a milder focal/grid laser treatment applied to the central retina reduced rates of moderate vision loss in eyes with DME by $50 \%$ over 3 years (51).

In the modern era, multiple phase 3 clinical trials have demonstrated the superiority of intravitreous anti-VEGF injections to laser monotherapy in reducing vision loss and improving rates of vision gain in eyes with DME (52-54). A recent comparative efficacy study of the 3 most commonly utilized anti-VEGF agents showed that all 3 agents - aflibercept, bevacizumab, and ranibizumab - were effective at improving vision over 1 and 2 years of treatment for $\operatorname{DME}(55,56)$. However, on average, treatment with aflibercept provided superior visual gains at 1 year as compared with bevacizumab and ranibizumab. Aflibercept remained superior to bevacizumab, but not ranibizumab, based on mean visual acuity outcomes after 2 years of therapy. Although first-line therapy for most eyes with central-involved DME consists of antiVEGF, intravitreous injections of steroid can also be effective for DME treatment $(57,58)$. However, intravitreous steroid use is limited by more frequent ocular side effects, such as cataract and glaucoma.

Anti-VEGF therapy is highly effective in regressing retinal neovascularization in eyes with PDR (59). Recent data suggest that anti-VEGF is a viable treatment alternative to PRP in eyes with PDR, especially for individuals with coexisting DME that already necessitates anti-VEGF therapy. Eyes treated with anti-VEGF for PDR have equivalent visual acuity outcomes at the 2-year endpoint of the study, compared with those treated with PRP. In addition, eyes treated with anti-VEGF exhibited better average visual acuity over the entire course of the 2-year study period (36). Additional benefits of anti-VEGF as compared with PRP include significantly less peripheral visual field loss, decreased rates of DME onset, and fewer vitrectomies over 2 years. Despite these benefits, anti-VEGF therapy may not be optimal for patients who cannot comply with the near-monthly follow-up and injection regimen required for adequate treatment and prevention of PDR recurrences.

Vitreoretinal surgery is utilized for cases of nonclearing vitreous hemorrhage from PDR or cases of PDR with tractional retinal detachment to relieve fibrous attachments that may be distorting the retina and causing vision loss or metamorphopsia (60). Vitrectomy with or without peeling of the internal limiting membrane can also be performed to treat DME, particularly when there is an epiretinal membrane or element of vitreoretinal traction leading to retinal thickening. Although retinal thickening is often improved after vitrectomy for DME, visual outcomes are less certain, with approximately a third of patients experiencing substantial visual improvement, but between 20\%-30\% experiencing substantial visual loss after surgery.

Although current therapies are effective at preventing vision loss and frequently result in visual gain for patients with both PDR and DME, unmet treatment needs still exist. A substantial proportion (40\%-50\%) of eyes with DME do not respond fully to anti-VEGF treatment, necessitating the development of novel therapies for this condition. For both PDR and DME, noninvasive, nondestructive, and longer-duration treatment options are also needed.

\section{The neurovascular unit: a framework for understanding DR}

Advances in understanding early cellular changes in the diabetic retina combined with improved retinal imaging have led to a conceptualization that DR can be viewed as a disease of the retinal neurovascular unit (Figure 2), which refers to the functional coupling and interdependency of neurons, glia, and vasculature (4) that integrate to regulate normal retinal function (61). An important facet of this integration is the coordination of local blood flow changes with fluctuations in metabolic demands. Retinal capillaries are composed of endothelial cells and pericytes but also have intimate associations with glial endfeet, neural processes, and professional immune cells such as microglia. Retinal arterioles have smooth muscle cells and, depending on the order of vessel, may also have significant pericyte coverage. These contractile cells respond dynamically to complex circulatory and neural cues to control blood flow (62). These cellular interactions are best recognized in the processes of neurovascular coupling, whereby neural, glial, and vascular cell interactions in both large and small vessels regulate blood flow to meet the metabolic demands of the retinal neuropile. This response is dysregulated in the diabetic retina prior 


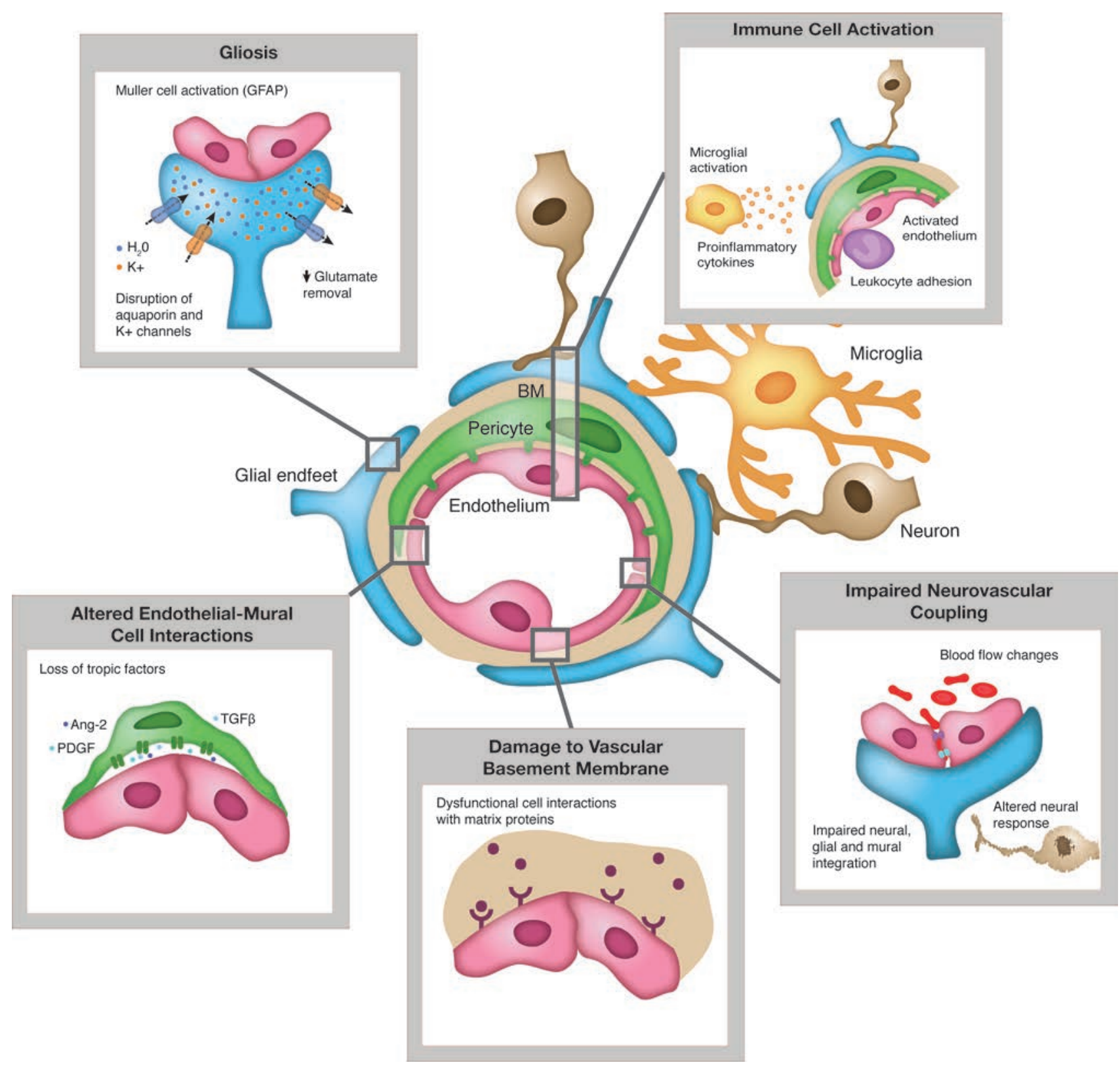

Figure 2. The neurovascular unit and its disruption by diabetes. In normal, healthy retina (shown in the center), there is functional coupling and interdependency of neurons, glial elements including Müller cells, and vascular cells, with associated immune cells such as microglia. The insets show pathological changes associated with diabetic retinopathy in multiple components of the neurovascular unit and interacting immune cells, including compromise of endothelial-mural cell interactions, vascular basement membrane damage, Müller cell gliosis, and immune cell activation. Together, these changes result in impairment of neurovascular coupling, with consequences including blood-retinal barrier breakdown and dysregulation of retinal blood flow. Illustrated by Rachel Davidowitz.

to appearance of observable vascular lesions, although it regulates the changes in blood flow that occur in animal models of DR (63) and in diabetic patients (64). Retinal vascular responses to diffuse illuminance flicker reflects impaired neurovascular coupling and abnormal endothelial-glia associations (65), resulting in attenuated arteriolar and venular dilatory responses (66) that may have early predictive value (67) in early-stage DR.

The conceptualization of DR as a disease of the neurovascular unit broadens our appreciation of the cell types that contribute to the development and progression of DR. Aside from the component vascular cells (endothelial cells and pericytes), diverse retinal neuronal cell types, macroglial elements (Müller cells and astrocytes), and microglia, the neurovascular concept also suggests the importance of additional cell types, such as immune cells and retinal pigment epithelium (RPE)/choroid, that impact the constituent 
cells of the neural retina. A greater understanding of the interactions of these various cellular elements and their pathogenic contributions could greatly expand the possibilities for new therapeutic strategies.

Pathology in the neural retina during $D R$. Vascular dysfunction and capillary loss are critical features of $\mathrm{DR}$, as evidenced by the impact on visual function stemming from treatments including anti-VEGF aimed at ameliorating retinal vascular changes. However, a growing body of evidence suggests that a neuropathy also exists in diabetic retina, perhaps even before overt nonperfusion of the neuropile. This broadening perspective has heightened the understanding of neuronal dysfunction and neurodegeneration and their corresponding clinical features, such as loss of color vision (68) and contrast sensitivity (69) and reduced electrical responses on electroretinographic testing $(70,71)$ that can occur before overt microvascular changes. Apoptotic death of retinal ganglion cells (RGC) and amacrine cells occurs in diabetic animal models (72) and has also been observed clinically in postmortem diabetic eyes $(73,74)$. Further evidence for structural changes include OCT imaging studies that demonstrate a reduction in thickness of the inner retinal layers in type 1 diabetics with minimal diabetic retinopathy $(75,76)$. Diabetes-induced alterations in the neurosensory retina could have major consequences, as neuronal dysfunction may contribute to the progression of vascular DR pathology. Retinal neurons, including photoreceptors, may be an important source of oxidative stress that help drive the proinflammatory environment in DR (77). In addition, retinal neuronal elements may secrete molecules, such as semaphorin $3 \mathrm{~A}$, that promote BRB dysfunction, contributing to macular edema (78). The notion that neuronal dysfunction and damage can promote clinical diabetic retinopathy, including microangiopathy, is supported by observational studies indicating that regional neuronal dysfunction ascertained by multifocal ERG predicts corresponding retinal locations that will develop retinopathy within $1-3$ years $(79,80)$. This potential pathogenic role for neurons has heightened interest in possible mechanisms for neuronal dysfunction and degeneration - including glutamate excitotoxicity, oxidative stress, and reduction of trophic support (81) - and prompted therapeutic strategies for DR based on neuroprotection $(82,83)$.

Glial dysfunction in DR. The astrocytes and Müller cell components of the neurovascular unit are impacted by diabetes, which alters the critical homeostatic function of these glia, especially relating to regulation of retinal blood flow, water balance in the neural parenchyma, and maintenance of barrier function (84). Specifically, Müller cells can undergo a reactive gliosis that is discernible by upregulation of glial acidic fibrilar protein (GFAP) (85) and increased expression of innate immune-related pathways reflected by proinflammatory cytokine secretion (86). Müller cell studies in a diabetic mouse model highlight a potential pivotal role of these cells in retinal vascular abnormalities in DR. Conditional KO mice with disrupted VEGF in Müller cells exhibited a decrease in biomarkers of retinal inflammation, including TNF- $\alpha$ and ICAM-1, as well as a reduction in retinal vascular abnormalities including leakage (87). This finding suggests that dysfunctional Müller cells could act in paracrine fashion to promote BRB dysfunction in DR. DR is also associated with the death of Müller cells (88), which further impacts the integrity of the neurovacular unit.

Immune cell function and inflammation in DR. Regulation of immune cells and control of inflammation is critical for the well-being and normal function of the retina. Resident microglial cells within the inner retina have a critical role in parainflammation: an adaptive response to tissue stress (including hyperglycemia and oxidative stress) and malfunction. While this response promotes homeostasis and normal tissue repair, in the short-term, chronic parainflammation contributes to initiation and progression of multiple disease processes (89). Within this context, the role of inflammation in driving the progression of DR is increasingly better appreciated (90, 91). As diabetes progresses, the retina exhibits multiple elements of chronic, subclinical inflammation, including immune cell activation and production of inflammatory molecules. Multiple immune cell types are activated in the early stages of DR, including enhanced leukocyte-endothelial interaction in the retinal vasculature (3, 90). This phenomenon of leukostasis is characterized by adherence of circulating myeloid cells, including neutrophils and monocytes, with activated vascular endothelium. Leukocyte-endothelial interactions can instigate damage to the retinal vascular endothelium and surrounding tissue both by physical occlusion of capillaries and through the release of inflammatory cytokines and superoxide. In addition to the contribution of intravascular immune cell types, activation of microglia as the resident immune cells of the retina and also infiltrating monocytes likely mediate the diabetes-induced generation of an inflammatory environment (3). Apart from activating professional immune cells, diabetes promotes a proinflammatory phenotype in other retinal cells, such as in the vasculature where upregulation of adhesion molecules including E-selectin and ICAM-1 $(92,93)$ enhance endothelial engagement with circulating immune cells. In addition, diabetes induces production of proinflammatory cytokines by Müller glia, including VEGF and TNF- $\alpha$ (87). Such events operate in concert to create an inflammatory milieu that contributes to the progression of DR. 
A key feature of DR is the increased expression of inflammatory cytokines and growth factors from various cell sources. For example, the proinflammatory peptide VEGF is well-recognized as a major player in DR, including its role in promoting retinal vascular permeability and DME (35). Strikingly, DME patients treated with anti-VEGF agents exhibited slower progression to capillary drop-out, suggesting that VEGF may play a role in progression of DR apart from its clearly documented effects on vascular permeability (94). Aside from VEGF, diabetes increases retinal levels of inflammatory cytokines including TNF- 0 and IL-1 $\beta$, both of which have been implicated in contributing to key pathologic endpoints in DR, including capillary drop-out and vascular permeability (95-97).

$R P E$ dysfunction and choroidopathy as components of $D R$. Although it has received comparatively less attention, diabetes also affects RPE function and leads to outer retinal changes that impact on photoreceptor and choroidal integrity. RPE culture-based models and in vivo studies have demonstrated that high glucose exposure or diabetes causes nitrosative stress (98) and metabolic changes relating to polyol metabolism (99). In the context of DME, new perspectives consider the contribution of changes to the outer BRB (oBRB) formed by the RPE. Disruption of normal oBRB function $(83,100)$ occurs during diabetes, and loss of RPE barrier properties leads to leakage of fluid from the choriocapillaris. The RPE also exhibits impaired fluid clearance from the retinal neuropile, which, in combination with loss of oBRB integrity, makes an important contribution to DME (101). The relative importance of the RPE dysfunction, as compared with retinal vascular leakage, in contributing to diabetic macular edema remains under active study.

The choriocapillaris itself incurs progressive damage during diabetes (as reviewed by Lutty; ref. 102). Diabetic choroidopathy occurs in patients (103) and animal models (104) and is manifest by thinning of the capillary bed with lesions such as vessel drop-out, aneurysms, ischemia, and in some cases intrachoroidal neovascularization $(103,105)$. Inflammatory cell infiltration may also participate in capillary occlusion and atrophy (106). Clinically, the diabetic choroid is beginning to receive more attention as imaging modalities improve. Beyond indocyanine green (ICG) angiographic studies (105), approaches such as enhanced depth imaging (EDI) have generally shown reduced choroidal thickness in diabetic eyes (107), although in some patient groups, increased thickness may occur (108) possibly relating to a postischemic fibrotic response and intrachoroidal neovascularization. In either case, choroidopathy in the diabetic eye could have a subsequent, profound impact on RPE and the outer retinal layers, which are oxygenated by the choriocapillaris. For example, the occurrence of basal laminar deposits (BLDs) in diabetic eyes are associated with areas of choriocapillaris degeneration (103). The precise nature of diabetic choroidopathy requires further clinical and experimental study, especially since this vascular bed is critical to normal retinal function.

\section{Future Directions}

Concept of protective mechanisms. While considerable research effort has been directed toward identifying pathogenic pathways contributing to initiation and progression of DR, a growing paradigm is the importance of endogenous mechanisms that protect against DR $(109,110)$. This concept is strongly supported by the Joslin 50-Year Medalist Study, which has enrolled over 1,000 individuals with T1D durations of 50 years or more. Approximately $50 \%$ of these patients have PDR, as might be expected given their long duration of diabetes. However, a substantial proportion (nearly $40 \%$ ) of the patients, all of whom were diagnosed with diabetes decades before strict glycemic control was standard practice, surprisingly still have no or mild DR (111). In contrast to other large studies in groups with shorter-duration diabetes, DR severity has not been associated with current or longitudinal $\mathrm{HbA} 1 \mathrm{c}$ values in this cohort, which suggests the presence of endogenous protective factors in those individuals who have not progressed to advanced diabetic complications, including retinopathy. The identification of such protective mechanisms might enable future novel therapies to prevent onset and early worsening of diabetic ocular disease. With the perspective that an imbalance between causal factors and protective factors governs progression of DR, new therapeutic strategies could center on stimulating the action of endogenous protective mechanisms. In this vein, several protective factors have been proposed in DR, including superoxide dismutase 2 (MnSOD) (112), pigment epithelium-derived factor (PEDF) (113), somatostatin (114), and NF-E2-related factor 2 (Nrf) (115). These molecules illustrate the potential benefit of promoting protective pathways in favorably modulating key processes in diabetic retinopathy. MnSOD and Nrf2 could serve to attenuate diabetes-induced oxidative stress. PEDF and Nrf2 counteract the proinflammatory environment in diabetic retinopathy. Somatostatin exerts a neuroprotective effect that could reduce neurodegeneration. An especially intriguing candidate protective factor for diabetic retinopathy is PPAR $\alpha$, which has been demonstrated to improve multiple beneficial endpoints in experimental models of DR, including inflammation and leakage (116, 
117). Strikingly, 2 large clinical trials of the PPAR $\alpha$ agonist fenofibrate showed an effect of this drug in slowing the progression of diabetic retinopathy. Although it is not clear whether this protective effect of fenofibrate was due to PPAR $\alpha$ agonism as opposed to its other potential actions, it is noteworthy that the protective effect was unrelated to the lipid-lowering activity of this drug (118). Pharmacologic targeting of these and other protective mechanisms therefore represent new therapeutic avenues in the treatment of DR.

New therapeutic angles in diabetic retinopathy. Although the past decade has seen significant improvements in the treatment options for DR, additional therapies are urgently required. Current therapies are directed exclusively toward advanced stages of DR, often after permanent damage has ensued; thus, treatments that are preventative or address early pathology are highly desirable. Anti-VEGF treatment is only partially effective against diabetic macular edema (119), and the identification of additional, VEGF-independent pathogenic molecules in this condition could lead to new treatments that better preserve vision. Additional molecular targets have been identified for pharmacologic inhibition, including TNF- $\alpha$ (120), the plasma kallikrein pathway (121), and lipoprotein-associated phospholipase A2 (Lp-PLA2) (122). Broadening therapeutic objectives beyond direct suppression of pathologic vascular changes may be promising. Notably, the expanded conceptualization of DR as a disease of the neurovascular unit brings to light additional cellular targets for therapy. With awareness that neuronal dysfunction and neurodegeneration are early events in DR, therapeutic strategies based on neuroprotection, including agents such as somatostatin, nerve growth factor (NGF), and brain-derived neurotrophic factor (BDNF) (114) are worthwhile to consider, and there is an ongoing clinical trial for early DR focused on retinal neuroprotection (82). Aside from neurons, the important role of Müller cells and microglia may direct efforts toward therapeutic targeting of these important cellular elements. Finally, expanding the scope of pathogenic processes that are therapeutically targeted beyond vascular leakage and neovascularization to include neuroprotection and intraretinal revascularization would be highly desirable endpoints in DR. The concept of epigenetic modifications associated with the metabolic memory phenomenon is an additional pathogenic process worthy of therapeutic attention (123). Identification and targeting of key epigenetic mechanisms could help to slow down the progression of DR in patients, especially those with a prior history of poor glycemic control. Finally, consideration of cell-based strategies including endothelial progenitor cells is worthwhile in promoting vascular repair and alleviating retinal ischemia (124).

In addition to the actual molecular and cellular targets that will require additional mechanistic insights, advances with respect to methods for administration of therapeutics will enable improvements in managing patients with DR. Intravitreal injections allow direct delivery of drugs to the retina with reduction of systemic side effects, but the necessity for frequent, repeat injections renders this approach appropriate only for more advanced and acute disease. Methods for topical or systemic delivery, as well as sustained release delivery methods including nanoparticles (3), would greatly aid treatment of chronic conditions such as diabetic macular edema. In addition, these strategies would also increase the feasibility of treating early stages of diabetic retinopathy, as the latter will require treatment over prolonged durations. Topical drug formulations that can reach the retina would reduce systemic side effects and allow self-administration by patients over long periods of time.

Precision medicine. Patients with diabetes exhibit great variation in the course of their retinopathy development, including both the pace of progression and the specific clinical manifestations. For instance, some individuals may have a stronger tendency to develop DME, whereas others may tend toward PDR. In addition, patients exhibit variable response to treatments; for instance, while most patients respond well to antiVEGF therapy, some have only a moderate or even poor response (125). Improvements in understanding the pathogenesis of the multiple facets of DR and the increase in diagnostic techniques, including imaging, opens the possibility of precision medicine geared toward directed strategies that take into account important differences between patients. Attaining greater understanding of patient variation and its impact on clinical phenotype will bolster efforts toward more precise management of DR. Such insights will likely arise from further mechanistic and biologic data, including genomics, proteomics, and biomarkers of disease. Together with advances in earlier diagnosis of DR, the objective of precision medicine is ideal in optimizing care of DR through improved phenotyping, understanding of mechanism, and expansion of treatment options.

\section{Conclusions}

Although the incidence of DR continues to increase, the past decade has seen the emergence of new treatment options, especially drugs targeting VEGF, which have greatly improved our management of DME and PDR endpoints. Nevertheless, a pressing need remains for efficacious new treatments for all stages of DR, and this underpins continuing efforts to fully understand the complex ways in which 
diabetes impacts the retina. An important conceptual advance has been the recognition that DR is a disease of the neurovascular unit, with multiple, interdependent cell types contributing to dysfunction of the retina. New therapeutic approaches should adopt this more holistic view of how diabetes affects the retina and tailor appropriate treatments to more precisely defined disease phenotypes with the exciting prospect of achieving successful clinical outcomes for all patients.

\section{Acknowledgments}

The authors would like to acknowledge financial support from the NIH NEI (EY022383 and EY022683), Fight for Sight (UK), The Sir Jules Thorn Trust, DEL/SFI, Wolfson Foundation, The Royal Society, NIH NEI R01 (EY024702), JDRF 3-SRA-2014-264-M-R, and Massachusetts Lions Eye Research Fund. The authors are grateful to $\mathrm{Zhenhua} \mathrm{Xu}$ for assistance with preparation of the figures.

Address correspondence to: Elia Duh, Department of Ophthalmology, Wilmer Ophthalmologic Institute, Johns Hopkins University School of Medicine, 400 N. Broadway, Room 3011, Baltimore, Maryland 21287, USA. Phone:1.410.614.3388; Email: eduh@jhmi.edu. Or to: Alan Stitt, Centre for Experimental Medicine, Queen's University Belfast, Belfast, BT9 7BL, Northern Ireland, United Kingdom. Phone: 44.28.9097.5375; Email: a.stitt@qub.ac.uk.

1. Guariguata L, Whiting DR, Hambleton I, Beagley J, Linnenkamp U, Shaw JE. Global estimates of diabetes prevalence for 2013 and projections for 2035. Diabetes Res Clin Pract. 2014;103(2):137-149.

2. Nanditha A, et al. Diabetes in Asia and the Pacific: Implications for the Global Epidemic. Diabetes Care. 2016;39(3):472-485.

3. Stitt AW, et al. The progress in understanding and treatment of diabetic retinopathy. Prog Retin Eye Res. 2016;51:156-186.

4. Antonetti DA, Klein R, Gardner TW. Diabetic retinopathy. N Engl J Med. 2012;366(13):1227-1239.

5. Klein R, Klein BE. Are individuals with diabetes seeing better?: a long-term epidemiological perspective. Diabetes. 2010;59(8):1853-1860.

6. Leasher JL, et al. Global Estimates on the Number of People Blind or Visually Impaired by Diabetic Retinopathy: A Meta-analysis From 1990 to 2010. Diabetes Care. 2016;39(9):1643-1649.

7. Cole ED, Novais EA, Louzada RN, Waheed NK. Contemporary retinal imaging techniques in diabetic retinopathy: a review. Clin Experiment Ophthalmol. 2016;44(4):289-299.

8. Jackson GR, Barber AJ. Visual dysfunction associated with diabetic retinopathy. Curr Diab Rep. 2010;10(5):380-384

9. Frey T, Antonetti DA. Alterations to the blood-retinal barrier in diabetes: cytokines and reactive oxygen species. Antioxid Redox Signal. 2011;15(5):1271-1284.

10. Zhang X, Zeng H, Bao S, Wang N, Gillies MC. Diabetic macular edema: new concepts in patho-physiology and treatment. Cell Biosci. 2014;4:27.

11. Diabetes Control Complications Trial Research Group, et al. The effect of intensive treatment of diabetes on the development and progression of long-term complications in insulin-dependent diabetes mellitus. N Engl J Med. 1993;329(14):977-986.

12. [No authors listed]. Effect of intensive blood-glucose control with metformin on complications in overweight patients with type 2 diabetes (UKPDS 34). UK Prospective Diabetes Study (UKPDS) Group. Lancet. 1998;352(9131):854-865.

13. Aiello LP, DCCT/EDIC Research Group. Diabetic retinopathy and other ocular findings in the diabetes control and complications trial/epidemiology of diabetes interventions and complications study. Diabetes Care. 2014;37(1):17-23.

14. Genuth S, et al. Skin advanced glycation end products glucosepane and methylglyoxal hydroimidazolone are independently associated with long-term microvascular complication progression of type 1 diabetes. Diabetes. 2015;64(1):266-278.

15. Zhong Q, Kowluru RA. Epigenetic changes in mitochondrial superoxide dismutase in the retina and the development of diabetic retinopathy. Diabetes. 2011;60(4):1304-1313.

16. Mishra M, Zhong Q, Kowluru RA. Epigenetic modifications of Keap1 regulate its interaction with the protective factor Nrf2 in the development of diabetic retinopathy. Invest Ophthalmol Vis Sci. 2014;55(11):7256-7265.

17. Reddy MA, Zhang E, Natarajan R. Epigenetic mechanisms in diabetic complications and metabolic memory. Diabetologia. 2015;58(3):443-455.

18. Friedrichs $\mathrm{P}$, et al. Hyperglycaemic memory affects the neurovascular unit of the retina in a diabetic mouse model. Diabetologia. 2017;60(7):1354-1358.

19. van Leiden HA, et al. Blood pressure, lipids, and obesity are associated with retinopathy: the hoorn study. Diabetes Care. 2002;25(8):1320-1325.

20. Yau JW, et al. Global prevalence and major risk factors of diabetic retinopathy. Diabetes Care. 2012;35(3):556-564

21. Mohamed Q, Gillies MC, Wong TY. Management of diabetic retinopathy: a systematic review. JAMA. 2007;298(8):902-916.

22. ACCORD Study Group, et al. Effects of medical therapies on retinopathy progression in type 2 diabetes. $N$ Engl J Med. 2010;363(3):233-244.

23. Hirsch IB, Brownlee M. Beyond hemoglobin A1c--need for additional markers of risk for diabetic microvascular complications. JAMA. 2010;303(22):2291-2292.

24. Klein R, Knudtson MD, Lee KE, Gangnon R, Klein BE. The Wisconsin Epidemiologic Study of Diabetic Retinopathy: XXII the twenty-five-year progression of retinopathy in persons with type 1 diabetes. Ophthalmology. 2008;115(11):1859-1868

25. Ljubimov AV, et al. Basement membrane abnormalities in human eyes with diabetic retinopathy. J Histochem Cytochem. 1996;44(12):1469-1479. 
26. COGAN DG, TOUSSAINT D, KUWABARA T. Retinal vascular patterns. IV. Diabetic retinopathy. Arch Ophthalmol. 1961;66:366-378

27. Yanoff M. Ocular pathology of diabetes mellitus. Am J Ophthalmol. 1969;67(1):21-38.

28. Mizutani M, Kern TS, Lorenzi M. Accelerated death of retinal microvascular cells in human and experimental diabetic retinopathy. J Clin Invest. 1996;97(12):2883-2890.

29. Gardiner TA, Archer DB, Curtis TM, Stitt AW. Arteriolar involvement in the microvascular lesions of diabetic retinopathy: implications for pathogenesis. Microcirculation. 2007;14(1):25-38.

30. Curtis TM, Gardiner TA, Stitt AW. Microvascular lesions of diabetic retinopathy: clues towards understanding pathogenesis? Eye (Lond). 2009;23(7):1496-1508.

31. Joyal JS, et al. Ischemic neurons prevent vascular regeneration of neural tissue by secreting semaphorin 3A. Blood. 2011;117(22):6024-6035.

32. Fukushima Y, et al. Sema3E-PlexinD1 signaling selectively suppresses disoriented angiogenesis in ischemic retinopathy in mice. J Clin Invest. 2011;121(5):1974-1985.

33. Wei Y, et al. Nrf2 in ischemic neurons promotes retinal vascular regeneration through regulation of semaphorin 6A. Proc Natl Acad Sci U S A. 2015;112(50):E6927-E6936.

34. Aiello LP, et al. Vascular endothelial growth factor in ocular fluid of patients with diabetic retinopathy and other retinal disorders. N Engl J Med. 1994;331(22):1480-1487.

35. El Rami H, Barham R, Sun JK, Silva PS. Evidence-Based Treatment of Diabetic Retinopathy. Semin Ophthalmol. 2017;32(1):67-74

36. Writing Committee for the Diabetic Retinopathy Clinical Research Network, et al. Panretinal Photocoagulation vs Intravitreous Ranibizumab for Proliferative Diabetic Retinopathy: A Randomized Clinical Trial. JAMA. 2015;314(20):2137-2146.

37. Khaliq A, et al. Increased expression of placenta growth factor in proliferative diabetic retinopathy. Lab Invest. 1998;78(1):109-116.

38. Butler JM, et al. SDF-1 is both necessary and sufficient to promote proliferative retinopathy. J Clin Invest. 2005;115(1):86-93.

39. Watanabe D, et al. Erythropoietin as a retinal angiogenic factor in proliferative diabetic retinopathy. $N$ Engl J Med. 2005;353(8):782-792.

40. [No authors listed]. Grading diabetic retinopathy from stereoscopic color fundus photographs--an extension of the modified Airlie House classification. ETDRS report number 10. Early Treatment Diabetic Retinopathy Study Research Group. Ophthalmology. 1991;98(5 Suppl):786-806.

41. Silva PS, et al. Peripheral Lesions Identified on Ultrawide Field Imaging Predict Increased Risk of Diabetic Retinopathy Progression over 4 Years. Ophthalmology. 2015;122(5):949-956.

42. Lammer J, et al. Cone Photoreceptor Irregularity on Adaptive Optics Scanning Laser Ophthalmoscopy Correlates With Severity of Diabetic Retinopathy and Macular Edema. Invest Ophthalmol Vis Sci. 2016;57(15):6624-6632.

43. Burns SA, et al. In vivo adaptive optics microvascular imaging in diabetic patients without clinically severe diabetic retinopathy. Biomed Opt Express. 2014;5(3):961-974.

44. Shin HJ, Lee SH, Chung H, Kim HC. Association between photoreceptor integrity and visual outcome in diabetic macular edema. Graefes Arch Clin Exp Ophthalmol. 2012;250(1):61-70.

45. Sun JK, et al. Disorganization of the retinal inner layers as a predictor of visual acuity in eyes with center-involved diabetic macular edema. JAMA Ophthalmol. 2014;132(11):1309-1316.

46. Sun JK, et al. Neural Retinal Disorganization as a Robust Marker of Visual Acuity in Current and Resolved Diabetic Macular Edema. Diabetes. 2015;64(7):2560-2570.

47. Bonnin S, Tadayoni R, Erginay A, Massin P, Dupas B. Correlation between ganglion cell layer thinning and poor visual function after resolution of diabetic macular edema. Invest Ophthalmol Vis Sci. 2015;56(2):978-982.

48. Agemy SA, et al. RETINAL VASCULAR PERFUSION DENSITY MAPPING USING OPTICAL COHERENCE TOMOGRAPHY ANGIOGRAPHY IN NORMALS AND DIABETIC RETINOPATHY PATIENTS. Retina (Philadelphia, Pa). 2015;35(11):2353-2363.

49. Harrison WW, et al. Prediction, by retinal location, of the onset of diabetic edema in patients with nonproliferative diabetic retinopathy. Invest Ophthalmol Vis Sci. 2011;52(9):6825-6831.

50. [No authors listed]. Preliminary report on effects of photocoagulation therapy. The Diabetic Retinopathy Study Research Group. Am J Ophthalmol. 1976;81(4):383-396.

51. [No authors listed]. Photocoagulation for diabetic macular edema. Early Treatment Diabetic Retinopathy Study report number 1. Early Treatment Diabetic Retinopathy Study research group. Arch Ophthalmol. 1985;103(12):1796-1806.

52. Diabetic Retinopathy Clinical Research Network, et al. Randomized trial evaluating ranibizumab plus prompt or deferred laser or triamcinolone plus prompt laser for diabetic macular edema. Ophthalmology. 2010;117(6):1064-1077.e35.

53. Brown DM, et al. Long-term outcomes of ranibizumab therapy for diabetic macular edema: the 36-month results from two phase III trials: RISE and RIDE. Ophthalmology. 2013;120(10):2013-2022.

54. Heier JS, et al. Intravitreal Aflibercept for Diabetic Macular Edema: 148-Week Results from the VISTA and VIVID Studies. Ophthalmology. 2016;123(11):2376-2385.

55. Diabetic Retinopathy Clinical Research Network, et al. Aflibercept, bevacizumab, or ranibizumab for diabetic macular edema. NEngl J Med. 2015;372(13):1193-1203.

56. Wells JA, et al. Aflibercept, Bevacizumab, or Ranibizumab for Diabetic Macular Edema: Two-Year Results from a Comparative Effectiveness Randomized Clinical Trial. Ophthalmology. 2016;123(6):1351-1359.

57. Campochiaro PA, et al. Sustained delivery fluocinolone acetonide vitreous inserts provide benefit for at least 3 years in patients with diabetic macular edema. Ophthalmology. 2012;119(10):2125-2132.

58. Boyer DS, et al. Three-year, randomized, sham-controlled trial of dexamethasone intravitreal implant in patients with diabetic macular edema. Ophthalmology. 2014;121(10):1904-1914.

59. Avery RL, et al. Intravitreal bevacizumab (Avastin) in the treatment of proliferative diabetic retinopathy. Ophthalmology. 2006;113(10):1695.e1-1695.15.

60. Berrocal MH, Acaba LA, Acaba A. Surgery for Diabetic Eye Complications. Curr Diab Rep. 2016;16(10):99.

61. Gardner TW, Davila JR. The neurovascular unit and the pathophysiologic basis of diabetic retinopathy. Graefes Arch Clin Exp 
Ophthalmol. 2017;255(1):1-6.

62. Kur J, Newman EA, Chan-Ling T. Cellular and physiological mechanisms underlying blood flow regulation in the retina and choroid in health and disease. Prog Retin Eye Res. 2012;31(5):377-406.

63. Mishra A, Newman EA. Inhibition of inducible nitric oxide synthase reverses the loss of functional hyperemia in diabetic retinopathy. Glia. 2010;58(16):1996-2004.

64. Nguyen TT, et al. Correlation of light-flicker-induced retinal vasodilation and retinal vascular caliber measurements in diabetes. Invest Ophthalmol Vis Sci. 2009;50(12):5609-5613.

65. Garhofer G, et al. Use of the retinal vessel analyzer in ocular blood flow research. Acta Ophthalmol. 2010;88(7):717-722.

66. Lim LS, et al. Dynamic responses in retinal vessel caliber with flicker light stimulation in eyes with diabetic retinopathy. Invest Ophthalmol Vis Sci. 2014;55(8):5207-5213.

67. Nguyen TT, et al. Flicker light-induced retinal vasodilation in diabetes and diabetic retinopathy. Diabetes Care. 2009;32(11):2075-2080.

68. Roy MS, Gunkel RD, Podgor MJ. Color vision defects in early diabetic retinopathy. Arch Ophthalmol. 1986;104(2):225-228.

69. Sokol S, Moskowitz A, Skarf B, Evans R, Molitch M, Senior B. Contrast sensitivity in diabetics with and without background retinopathy. Arch Ophthalmol. 1985;103(1):51-54.

70. Gardner TW, Abcouwer SF, Barber AJ, Jackson GR. An integrated approach to diabetic retinopathy research. Arch Ophthalmol. 2011;129(2):230-235.

71. Abcouwer SF, Gardner TW. Diabetic retinopathy: loss of neuroretinal adaptation to the diabetic metabolic environment. Ann $N$ Y Acad Sci. 2014;1311:174-190.

72. Barber AJ, Lieth E, Khin SA, Antonetti DA, Buchanan AG, Gardner TW. Neural apoptosis in the retina during experimental and human diabetes. Early onset and effect of insulin. J Clin Invest. 1998;102(4):783-791.

73. Carrasco E, Hernández C, Miralles A, Huguet P, Farrés J, Simó R. Lower somatostatin expression is an early event in diabetic retinopathy and is associated with retinal neurodegeneration. Diabetes Care. 2007;30(11):2902-2908.

74. Barber AJ, Lieth E, Khin SA, Antonetti DA, Buchanan AG, Gardner TW. Neural apoptosis in the retina during experimental and human diabetes. Early onset and effect of insulin. J Clin Invest. 1998;102(4):783-791.

75. van Dijk HW, et al. Selective loss of inner retinal layer thickness in type 1 diabetic patients with minimal diabetic retinopathy. Invest Ophthalmol Vis Sci. 2009;50(7):3404-3409.

76. van Dijk HW, et al. Decreased retinal ganglion cell layer thickness in patients with type 1 diabetes. Invest Ophthalmol Vis Sci. 2010;51(7):3660-3665.

77. Du Y, Veenstra A, Palczewski K, Kern TS. Photoreceptor cells are major contributors to diabetes-induced oxidative stress and local inflammation in the retina. Proc Natl Acad Sci U S A. 2013;110(41):16586-16591.

78. Cerani A, et al. Neuron-derived semaphorin $3 \mathrm{~A}$ is an early inducer of vascular permeability in diabetic retinopathy via neuropilin-1. Cell Metab. 2013;18(4):505-518.

79. Han Y, Adams AJ, Bearse MA, Schneck ME. Multifocal electroretinogram and short-wavelength automated perimetry measures in diabetic eyes with little or no retinopathy. Arch Ophthalmol. 2004;122(12):1809-1815.

80. Harrison WW, et al. Multifocal electroretinograms predict onset of diabetic retinopathy in adult patients with diabetes. Invest Ophthalmol Vis Sci. 2011;52(2):772-777.

81. Barber AJ, Gardner TW, Abcouwer SF. The significance of vascular and neural apoptosis to the pathology of diabetic retinopathy. Invest Ophthalmol Vis Sci. 2011;52(2):1156-1163.

82. Hernández C, Simó-Servat O, Simó R. Somatostatin and diabetic retinopathy: current concepts and new therapeutic perspectives. Endocrine. 2014;46(2):209-214.

83. Omri S, et al. PKC $\zeta$ mediates breakdown of outer blood-retinal barriers in diabetic retinopathy. PLoS One. 2013;8(11):e81600.

84. Bringmann A, Wiedemann P. Müller glial cells in retinal disease. Ophthalmologica. 2012;227(1):1-19.

85. Hammes HP, Federoff HJ, Brownlee M. Nerve growth factor prevents both neuroretinal programmed cell death and capillary pathology in experimental diabetes. Mol Med. 1995;1(5):527-534.

86. Zong H, et al. Hyperglycaemia-induced pro-inflammatory responses by retinal Müller glia are regulated by the receptor for advanced glycation end-products (RAGE). Diabetologia. 2010;53(12):2656-2666.

87. Wang J, Xu X, Elliott MH, Zhu M, Le YZ. Müller cell-derived VEGF is essential for diabetes-induced retinal inflammation and vascular leakage. Diabetes. 2010;59(9):2297-2305.

88. Feenstra DJ, Yego EC, Mohr S. Modes of Retinal Cell Death in Diabetic Retinopathy. J Clin Exp Ophthalmol. 2013;4(5):298.

89. Xu H, Chen M, Forrester JV. Para-inflammation in the aging retina. Prog Retin Eye Res. 2009;28(5):348-368.

90. Tang J, Kern TS. Inflammation in diabetic retinopathy. Prog Retin Eye Res. 2011;30(5):343-358.

91. Zhang W, Liu H, Rojas M, Caldwell RW, Caldwell RB. Anti-inflammatory therapy for diabetic retinopathy. Immunotherapy. 2011;3(5):609-628.

92. McLeod DS, Lefer DJ, Merges C, Lutty GA. Enhanced expression of intracellular adhesion molecule-1 and P-selectin in the diabetic human retina and choroid. Am J Pathol. 1995;147(3):642-653.

93. Hartsock MJ, Cho H, Wu L, Chen WJ, Gong J, Duh EJ. A Mouse Model of Retinal Ischemia-Reperfusion Injury Through Elevation of Intraocular Pressure. J Vis Exp. 2016;(113):e54065.

94. Miller WP, et al. The Translational Repressor 4E-BP1 Contributes to Diabetes-Induced Visual Dysfunction. Invest Ophthalmol Vis Sci. 2016;57(3):1327-1337.

95. Vincent JA, Mohr S. Inhibition of caspase-1/interleukin-1beta signaling prevents degeneration of retinal capillaries in diabetes and galactosemia. Diabetes. 2007;56(1):224-230.

96. Kowluru RA, Odenbach S. Role of interleukin-1beta in the development of retinopathy in rats: effect of antioxidants. Invest Ophthalmol Vis Sci. 2004;45(11):4161-4166.

97. Joussen AM, et al. Nonsteroidal anti-inflammatory drugs prevent early diabetic retinopathy via TNF-alpha suppression. FASEB J. 2002;16(3):438-440.

98. Rosales MA, Silva KC, Duarte DA, Rossato FA, Lopes de Faria JB, Lopes de Faria JM. Endocytosis of tight junctions caveolin nitrosylation dependent is improved by cocoa via opioid receptor on RPE cells in diabetic conditions. Invest Ophthalmol Vis Sci. 2014;55(9):6090-6100. 
99. Samuels IS, Lee CA, Petrash JM, Peachey NS, Kern TS. Exclusion of aldose reductase as a mediator of ERG deficits in a mouse model of diabetic eye disease. Vis Neurosci. 2012;29(6):267-274.

100. Beasley S, et al. Caspase-14 expression impairs retinal pigment epithelium barrier function: potential role in diabetic macular edema. Biomed Res Int. 2014;2014:417986.

101. Simó R, Villarroel M, Corraliza L, Hernández C, Garcia-Ramírez M. The retinal pigment epithelium: something more than a constituent of the blood-retinal barrier--implications for the pathogenesis of diabetic retinopathy. J Biomed Biotechnol. 2010;2010:190724.

102. Lutty GA. Effects of diabetes on the eye. Invest Ophthalmol Vis Sci. 2013;54(14):ORSF81-ORSF87.

103. Cao J, McLeod S, Merges CA, Lutty GA. Choriocapillaris degeneration and related pathologic changes in human diabetic eyes Arch Ophthalmol. 1998;116(5):589-597.

104. Muir ER, Rentería RC, Duong TQ. Reduced ocular blood flow as an early indicator of diabetic retinopathy in a mouse model of diabetes. Invest Ophthalmol Vis Sci. 2012;53(10):6488-6494.

105. Hua R, Liu L, Wang X, Chen L. Imaging evidence of diabetic choroidopathy in vivo: angiographic pathoanatomy and choroidal-enhanced depth imaging. PLoS One. 2013;8(12):e83494

106. Lutty GA, Cao J, McLeod DS. Relationship of polymorphonuclear leukocytes to capillary dropout in the human diabetic choroid. Am J Pathol. 1997;151(3):707-714.

107. Querques G, et al. Enhanced depth imaging optical coherence tomography in type 2 diabetes. Invest Ophthalmol Vis Sci. 2012;53(10):6017-6024

108. Kim JT, Lee DH, Joe SG, Kim JG, Yoon YH. Changes in choroidal thickness in relation to the severity of retinopathy and macular edema in type 2 diabetic patients. Invest Ophthalmol Vis Sci. 2013;54(5):3378-3384.

109. Rask-Madsen C, King GL. Kidney complications: factors that protect the diabetic vasculature. Nat Med. $2010 ; 16(1): 40-41$.

110. Rask-Madsen C, King GL. Vascular complications of diabetes: mechanisms of injury and protective factors. Cell Metab. 2013;17(1):20-33.

111. Sun JK, et al. Protection from retinopathy and other complications in patients with type 1 diabetes of extreme duration: the joslin 50-year medalist study. Diabetes Care. 2011;34(4):968-974

112. Kowluru RA, Atasi L, Ho YS. Role of mitochondrial superoxide dismutase in the development of diabetic retinopathy. Invest Ophthalmol Vis Sci. 2006;47(4):1594-1599.

113. Zhang SX, Wang JJ, Gao G, Shao C, Mott R, Ma JX. Pigment epithelium-derived factor (PEDF) is an endogenous antiinflammatory factor. FASEB J. 2006;20(2):323-325.

114. Simó R, Hernández C, European Consortium for the Early Treatment of Diabetic Retinopathy (EUROCONDOR). Neurodegeneration in the diabetic eye: new insights and therapeutic perspectives. Trends Endocrinol Metab. 2014;25(1):23-33.

115. Xu Z, et al. NRF2 plays a protective role in diabetic retinopathy in mice. Diabetologia. 2014;57(1):204-213.

116. Chen Y, et al. Therapeutic effects of PPAR $\alpha$ agonists on diabetic retinopathy in type 1 diabetes models. Diabetes. 2013;62(1):261-272.

117. $\mathrm{Hu}$ Y, et al. Pathogenic role of diabetes-induced PPAR- $\alpha$ down-regulation in microvascular dysfunction. Proc Natl Acad Sci U S A. 2013;110(38):15401-15406

118. Noonan JE, Jenkins AJ, Ma JX, Keech AC, Wang JJ, Lamoureux EL. An update on the molecular actions of fenofibrate and its clinical effects on diabetic retinopathy and other microvascular end points in patients with diabetes. Diabetes. 2013;62(12):3968-3975.

119. Ford JA, Lois N, Royle P, Clar C, Shyangdan D, Waugh N. Current treatments in diabetic macular oedema: systematic review and meta-analysis. BMJ Open. 2013;3(3):e002269.

120. Sfikakis PP, et al. Infliximab for diabetic macular edema refractory to laser photocoagulation: a randomized, double-blind, placebo-controlled, crossover, 32-week study. Diabetes Care. 2010;33(7):1523-1528.

121. Liu J, Feener EP. Plasma kallikrein-kinin system and diabetic retinopathy. Biol Chem. 2013;394(3):319-328

122. Canning P, et al. Lipoprotein-associated phospholipase A2 (Lp-PLA2) as a therapeutic target to prevent retinal vasopermeability during diabetes. Proc Natl Acad Sci U S A. 2016;113(26):7213-7218.

123. Kowluru RA, Kowluru A, Mishra M, Kumar B. Oxidative stress and epigenetic modifications in the pathogenesis of diabetic retinopathy. Prog Retin Eye Res. 2015;48:40-61.

124. Shaw LC, Neu MB, Grant MB. Cell-based therapies for diabetic retinopathy. Curr Diab Rep. 2011;11(4):265-274.

125. Ashraf M, Souka A, Adelman R. Predicting outcomes to anti-vascular endothelial growth factor (VEGF) therapy in diabetic macular oedema: a review of the literature. Br J Ophthalmol. 2016;100(12):1596-1604. 